\title{
COMMENTS
}

Comments are short papers which criticize or correct papers of other authors previously published in the Physical Review. Each Comment should state clearly to which paper it refers and must be accompanied by a brief abstract. The same publication schedule as for regular articles is followed, and page proofs are sent to authors.

\section{Comment on "Nonlinear resonance and chaos in the relativistic phase space for driven nonlinear systems"}

\author{
D. G. Luchinsky, P. V. E. McClintock, S. M. Soskin, ${ }^{*}$ and N. D. Stein \\ School of Physics and Chemistry, Lancaster University, Lancaster, LA1 4YB, United Kingdom
}

\author{
A. B. Neiman
}

Department of Physics, Saratov State University, Saratov, Russia

(Received 18 September 1995)

\begin{abstract}
Kim and Lee [Phys. Rev. E 52, 473 (1995)] report relativity-induced resonances in periodically driven oscillators. We comment that zero-dispersion nonlinear resonance (ZDNR) will occur in some of the systems considered, outline the physical origins of the ZDNR, and propose an explanation of a discrepancy noted by Kim and Lee between their theoretical and numerical values of the energy at the stationary stable points of Poincaré sections.

PACS number(s): 05.45. $+\mathrm{b}, 03.20 .+\mathrm{i}$
\end{abstract}

In a recent paper [1], Kim and Lee have reported some very interesting phenomena in the relativistic dynamics of periodically driven oscillators. They pointed out that the dependence on energy $E$ of a frequency of eigenoscillation $\Omega(E)$ could acquire a local maximum at high energies-a purely relativistic effect-leading to the appearance of resonances that were absent in the nonrelativistic description. We should like to comment that while the simple resonance analysis of [1] provides a good approximation under most conditions, it fails when the dispersion $d \Omega(E) / d E$ is very small, e.g., close to an extremum in $\Omega(E)$, such as the relativity-induced maximum reported in [1].

An approach applicable to the case of very small dispersion has, however, recently been developed [2]. It has led to the identification of zero-dispersion nonlinear resonance (ZDNR) and related phenomena that occur in the close vicinity of extrema in $\Omega(E)$. ZDNR differs in several important respects [2-5] from conventional nonlinear resonance (NR), including in the topology of its phase space, and it can occur even when the analysis used in [1] would not predict any resonances at all [2].

An illustration of the topological change that occurs at the ZDNR-NR transition for the driven double-well Duffing oscillator of [1] is shown in Fig. 1: compare Fig. 1(a), which reproduces in more detail Fig. 2(a) of [1], with Fig. 1(b), which corresponds to the same frequency but with a larger driving force amplitude. In the NR stage [Fig. 1(a)], the separatrix of the left resonance lies inside the separatrix of the right one, whereas it is the other way round for ZDNR, as

\footnotetext{
* Permanent address: Institute of Semiconductor Physics, Ukrainian National Academy of Sciences, Kiev, Ukraine.
}

can be seen from Fig. 1(b). Besides this, for trajectories trapped in nonlinear resonance, the difference of the limiting values between which the slow phase (angle) oscillates approaches $2 \pi$ in the case of NR, as seen in Fig. 1(a). For ZDNR, on the other hand, this difference is smaller [Fig. $1(b)]$ and tends to zero as $F_{0}$ grows. Apart from differences in their pure dynamics, the distinctions between ZDNR and NR can be manifested very strongly in the presence of fluctuations [4].

Although the analysis and discussion of [2-5] were introduced with classical systems in mind, it is clear that zerodispersion phenomena including ZDNR also occur for some of the relativistic systems considered in [1]. The phenomena can be described in terms of slow variables, action $I$ and phase $\tilde{\psi}$, that average over the high-frequency oscillations [6]; the term proportional to the first power of the amplitude of the driving force is retained in the expression for $\dot{\tilde{\psi}}$. For the period- $n$ resonance, using the notation of [1] [energy $E$, action $I=I(E)$, driving force frequency and amplitude $\omega$ and $F_{0}$ respectively], the dynamical equations can be written (cf. [2])

$$
\dot{I}=-\frac{\partial \tilde{H}}{\partial \tilde{\psi}}, \quad \dot{\tilde{\psi}}=\frac{\partial \tilde{H}}{\partial I}, \quad \tilde{H}=n E-\omega I+n F_{0} q_{n} \cos (\tilde{\psi}),
$$

where the $q_{n}$ are coefficients in the Fourier expansion of $q \equiv q(E, \psi)=2 \sum_{n=0}^{\infty} q_{n}(E) \cos (n \psi)$. Stationary solutions $\tilde{\psi}_{\mathrm{st}}$ can be either stable or unstable and can be of two types: with $\tilde{\psi}_{\mathrm{st}}=0$ or $\tilde{\psi}_{\mathrm{st}}=\pi$. The stationary energies $E_{\mathrm{st}}$ are derived from

$$
1-\frac{\omega}{n \Omega}= \pm F_{0} \frac{d q_{n}}{d E}
$$



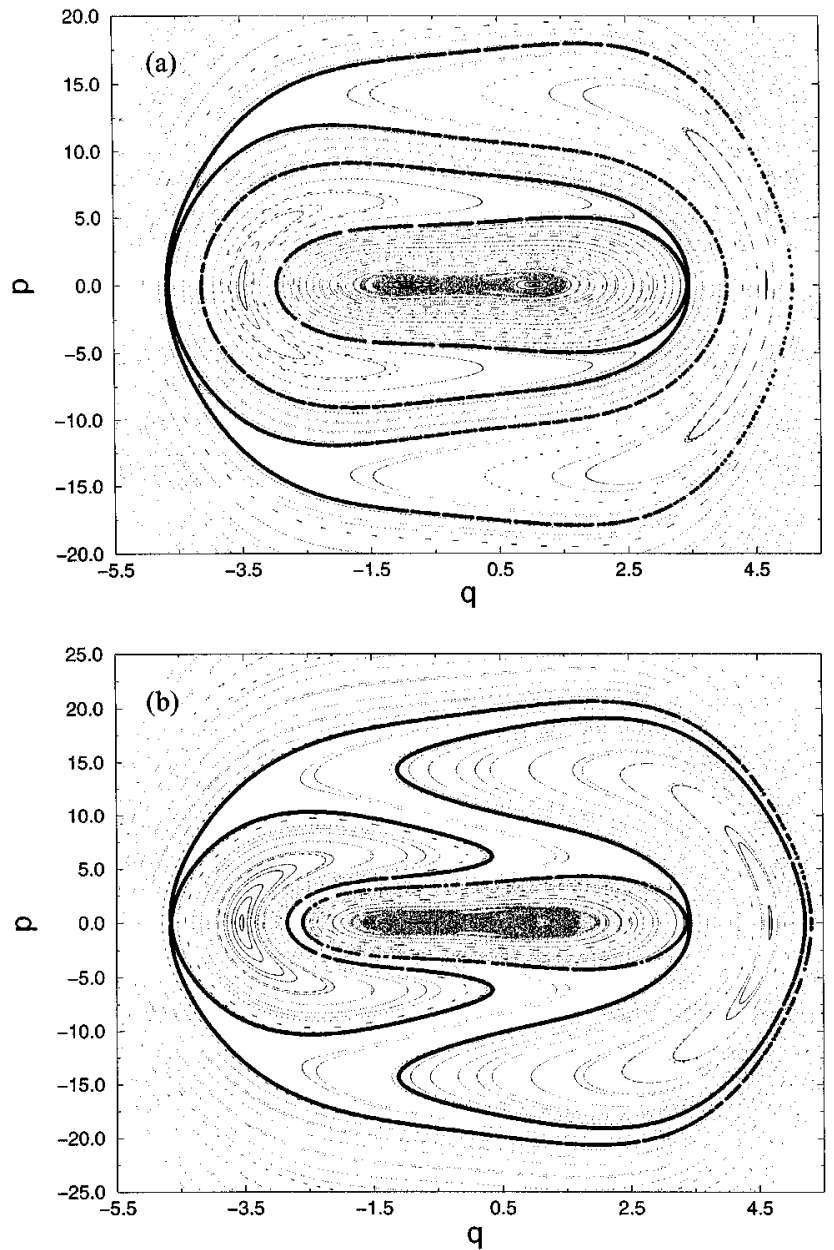

FIG. 1. Poincaré sections for the driven double-well Duffing oscillator considered in [1]: (a) for the same parameters as Fig. 2(b) of [1], with $F_{0}=0.1$, and (b) with $F_{0}=0.3$. The separatrices, separating nonlinear resonances (regions for which the energy oscillates in a narrow band about the resonant energy and the phase oscillates within finite limits) from other regions of the phase space, are indicated by larger dots (the gaps are due to a finite integration time).

where $\Omega \equiv \Omega(E)$ is a frequency of eigenoscillation and the plus and minus correspond, respectively, to $\tilde{\psi}_{\mathrm{st}}=\pi$ and $\psi_{\mathrm{st}}=0$.

Large energy solutions of (2) for the same parameters as in Fig. 1(b) are shown in Fig. 2. Unstable solutions correspond to saddle points of $\tilde{H}(1)$ and the ZDNR-NR transition

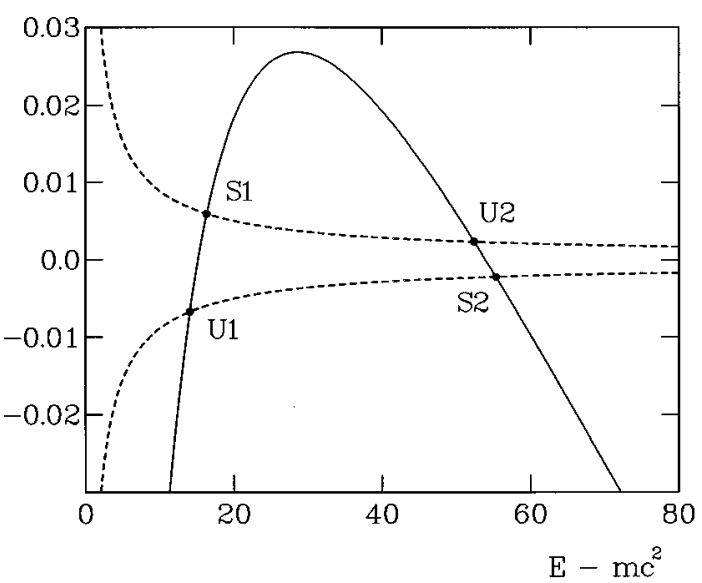

FIG. 2. Quantities $1-\omega / \Omega$ (full line) and $\pm F_{0}\left(d q_{1} / d E\right.$ ) (dashed lines) plotted as functions of energy $E$ for the same parameters as in Fig. 1(b). Their intersections, corresponding to stable and unstable stationary solutions of (2) in the range of large energies, are labeled $S 1, S 2$ and $U 1, U 2$, respectively.

occurs [5] when the $\tilde{H}$ values at the saddle points become equal to each other. If the resonances lie close enough to the local maximum of $\Omega(E)$, where a parabolic approximation is valid, the value of $F_{0}$ at which the ZDNR-NR transition occurs (cf. [5]) is

$$
F_{0}=\frac{1}{3 q_{n}\left(E_{m}\right)}\left(\frac{8 n \Omega\left(E_{m}\right)}{\left|d^{2} \Omega\left(E_{m}\right) / d E_{m}^{2}\right|}\right)^{1 / 2}\left(1-\frac{\omega}{n \Omega\left(E_{m}\right)}\right)^{3 / 2},
$$

where $E_{m}$ is the energy of the maximum of $\Omega(E)$. For the parameter values of Fig. 1, the transition occurs at $F_{0}=0.19$. Note also that the stable points do not coincide with exact resonances, i.e., with intersections of the full curve with the horizontal zero level in Fig. 2. This probably accounts for the discrepancy of up to $20 \%$ reported in [1] between the theoretical and numerical values of the energy at the stationary stable points of the Poincaré sections.

In conclusion, we comment that zero-dispersion phenomena may be expected to occur not only in some of the model relativistic systems considered in [1], but also in certain real relativistic systems such as driven electron plasma waves [7] and periodically focused intense beams of charged particles [8].
[1] J.-H. Kim and H.-W. Lee, Phys. Rev. E 52, 473 (1995).

[2] S. M. Soskin, Phys. Rev. E 50, R44 (1994).

[3] A. B. Neiman, S. M. Soskin, and P. V. E. McClintock, in Noise in Physical Systems and $1 / f$ Fluctuations, edited by V. Bareikis and R. Katilius (World Scientific, Singapore, 1995), p. 701.

[4] D. G. Luchinsky, P. V. E. McClintock, S. M. Soskin, and R. Mannella, in Noise in Physical Systems and $1 / f$ Fluctuations (Ref. [3]), p. 705.
[5] S. M. Soskin and D. G. Luchinsky, Nuovo Cimento D 17, 915 (1995).

[6] G. M. Zaslavsky, R. Z. Sagdeev, D. A. Usikov, and A. A. Chernikov, Weak Chaos and Quasi-Regular Patterns (Cambridge University Press, Cambridge, 1991).

[7] W. P. Leemans, C. Joshi, W. B. Mori, C. E. Clayton, and T. W. Johnston, Phys. Rev. A 46, 5112 (1992).

[8] C. Chen and R. C. Davidson, Phys. Rev. Lett. 72, 2195 (1994). 